\title{
Identifying the counterpart of HESS J1858+020
}

\author{
S. Paron and E. Giacani \\ Instituto de Astronomía y Física del Espacio (CONICET-UBA), CC 67, Suc. 28, 1428 Buenos Aires, Argentina \\ e-mail: sparon@iafe.uba.ar
}

Received 18 November 2009 / Accepted 14 December 2009

ABSTRACT

\begin{abstract}
Aims. HESS J1858+020 is a weak $\gamma$-ray source that does not have any clear cataloged counterpart at any wavelengths. Recently, the source G35.6-0.4 was re-identified as a SNR. The HESS source lies towards the southern border of this remnant. The purpose of this work is to investigate the interstellar medium around the mentioned sources to look for possible counterparts of the very high energy emission.

Methods. Using the ${ }^{13} \mathrm{CO} J=1-0$ line from the Galactic Ring Survey and mid-IR data from GLIMPSE we analyze the environs of HESS J1858+020 and SNR G35.6-0.4.

Results. The ${ }^{13} \mathrm{CO}$ data show the presence of a molecular cloud towards the southern border of SNR G35.6-0.4 and at the same distance as the remnant. This cloud is composed of two molecular clumps, one over the SNR shell and the other located at the center of HESS J1858+020. We estimate a molecular mass and a density of $\sim 5 \times 10^{3} M_{\odot}$ and $\sim 500 \mathrm{~cm}^{-3}$ respectively for each clump. Considering the gamma-ray flux observed towards HESS J1858+020, we estimate that a molecular cloud with a density of at least $150 \mathrm{~cm}^{-3}$ could explain the very high energy emission hadronically. Thus, we suggest that the $\gamma$-ray emission detected in HESS J1858+020 is due to hadronic mechanism. Additionally, analyzing mid-IR emission, we find that the region is active in star formation, which could be considered as an alternative or complementary possibility to explain the very high energy emission.
\end{abstract}

Key words. ISM: clouds - ISM: supernova remnants - gamma rays: ISM - ISM: individual objects: HESS J1858+020

\section{Introduction}

Among the rich population of $\mathrm{TeV} \gamma$-ray sources, several are associated with Galactic phenomena which include supernova remnants (SNRs), pulsar wind nebulae (PWNe) and binary systems (Hinton 2008). Extragalactic TeV sources are associated with active AGNs (mostly blazars). There are however a number of $\gamma$-ray sources which lack as yet a clear identification or an established origin. In this paper we focus on the very high energy source HESS J1858+020.

HESS J1858+020 is a weak $\gamma$-ray source detected with HESS (the High Energy Stereoscopic System), an array of air Cherenkov telescopes. Though it is a nearly point-like source, its morphology shows a slight extension of $\sim 5^{\prime}$ along its major axis. The source has been detected at a significance level of $7 \sigma$ with a differential spectral index of $2.2 \pm 0.1$. At the present, HESS J1858+020 does not have any clear cataloged counterpart at any wavelengths. The pulsar PSR J1858+0143, which is energetic enough to power HESS J1858+020, is located far away from the center of the gamma source, thus an association between them is unlikely (Aharonian et al. 2008a).

Recently Green (2009), using radio continuum data from the Very Large Array (VLA) Galactic Plane Survey (VGPS, Stil et al. 2006), re-identified the radio continuum source G35.6-0.4 as a supernova remnant (SNR). This remnant is seen in projection over the northern border of HESS J1858+020. Green (2009) estimated an age of 30000 years for the SNR and a distance of $\sim 10.5 \mathrm{kpc}$ based on the proximity of the remnant with the HII region G35.5-0.0. As pointed out in several works (see Aharonian \& Atoyan 1996; Yamazaki et al. 2006; Gabici et al. 2007, 2009), an SNR, in particular an old remnant (i.e. age larger than a few 10000 years), interacting with a molecular cloud could explain the origin of the $\gamma$-ray emission via pion decay from protonproton collisions. In this context, we expect a correlation between $\gamma$-ray emission and matter concentration.

In this letter we study the interstellar medium around the sources G35.6-0.4 and HESS J1858+020 to investigate the possible origin for the very high energy emission.

\section{Data}

To analyze the ISM towards HESS J1858+020 we used the ${ }^{13} \mathrm{CO}$ $J=1-0$ emission obtained from the Galactic Ring Survey. This survey maps the Galactic Ring with an angular and spectral resolution of $46^{\prime \prime}$ and $0.2 \mathrm{~km} \mathrm{~s}^{-1}$, respectively (see Jackson et al. 2006). We also used the mosaiced images from GLIMPSE and MIPSGAL and the GLIMPSE Point-Source Catalog (GPSC) in the Spitzer-IRAC $(3.6,4.5,5.8$ and $8 \mu \mathrm{m})$. IRAC has an angular resolution between 1'.5 and 1.'9 (see Fazio et al. 2004; Werner et al. 2004). MIPSGAL is a survey of the same region as GLIMPSE, using the MIPS instrument $(24$ and $70 \mu \mathrm{m})$ on Spitzer. The MIPSGAL resolution at $24 \mu \mathrm{m}$ is $6^{\prime \prime}$.

\section{Results and discussion}

Analyzing the whole ${ }^{13} \mathrm{CO} J=1-0$ data cube in a region containing SNR G25.6-0.4 and HESS J1858+020, we find a molecular cloud lying in the southern border of the remnant in the velocity range between 51 and $59 \mathrm{~km} \mathrm{~s}^{-1}$. This velocity range coincides with the systemic velocity of G25.6-0.4 derived from its estimated distance (Green 2009). Figure 1 shows a three color image where red represents the Spitzer-IRAC $8 \mu \mathrm{m}$ band, green 


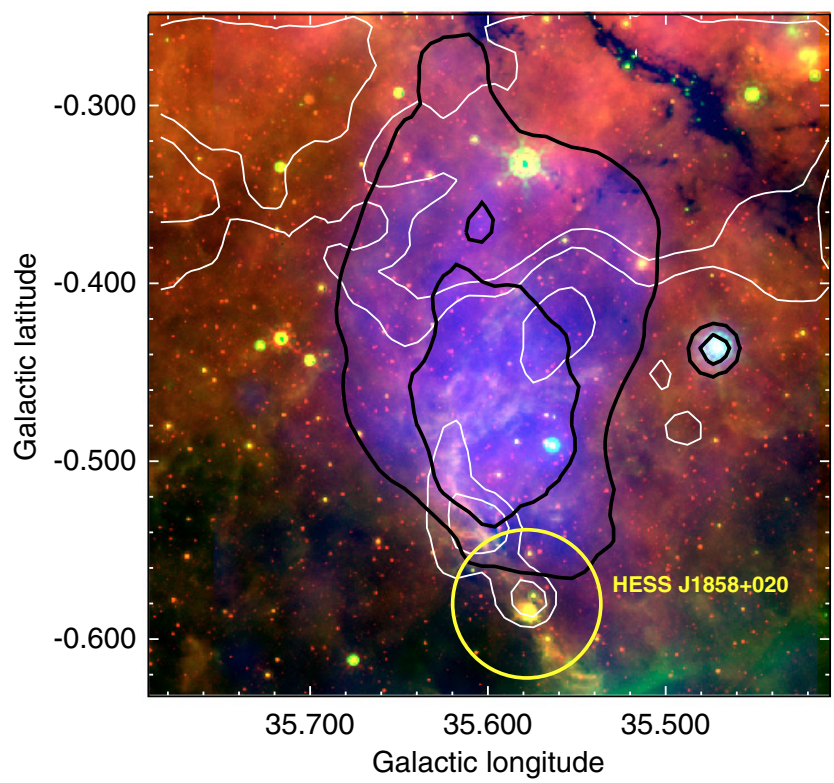

Fig. 1. Three color image of the analyzed region (red $=8 \mu \mathrm{m}$, green $=24 \mu \mathrm{m}$, and blue $=20 \mathrm{~cm}$ radio continuum emission). The black contours delineate the radio continuum emission with levels of 20 and $28 \mathrm{~K}$. The white contours are the ${ }^{13} \mathrm{CO} J=1-0$ emission integrated between 51 and $59 \mathrm{~km} \mathrm{~s}^{-1}$, its levels are 3.2 and $5 \mathrm{~K} \mathrm{~km} \mathrm{~s}^{-1}$. The yellow circle represents the source HESS J1858+020.

is the MIPSGAL emission at $24 \mu \mathrm{m}$ and blue is the radio continuum emission of G35.6-0.4 at $20 \mathrm{~cm}$ from the VGPS. Black contours delineate this last emission, and white contours are the ${ }^{13} \mathrm{CO} J=1-0$ emission integrated between 51 and $59 \mathrm{~km} \mathrm{~s}^{-1}$. As mentioned above, the extension of the HESS source is slightly elliptical with $\sim 5^{\prime}$ along its major axis. As the extent and angle are not well constrained, we represent in Fig. 1 the position and the extension of HESS J1858+020 with a circle of $5^{\prime}$ in diameter. Can be seen from this figure that the molecular cloud presents two well-defined clumps, one centered at $l=35^{\circ} .60, b=-0.53$ over the SNR shell, and the other centered at $l=35.58, b=-0.58$, in coincidence with the center of HESS J1858+020. The $\gamma$-ray emission could be related to both molecular clumps.

By inspecting the ${ }^{13} \mathrm{CO}$ spectra we found that the clump located over the SNR shell shows some possible kinematical evidence of shocked gas. As Fig. 2 displays, the spectra are not symmetric and present a slight spectral shoulder or a less intense component at "blueshifted" velocities. It could be evidence of turbulent motion in the gas, may be produced by the SNR shock (see e.g. Falgarone et al. 1994). Taking into account the positional and kinematical agreement between the molecular gas and the SNR G35.6-0.4, we suggest that the remnant is interacting with the adjacent molecular cloud.

Using the ${ }^{13} \mathrm{CO} J=1-0$ line and assuming local thermodynamic equilibrium (LTE) we estimate the $\mathrm{H}_{2}$ column density towards the molecular clumps described above. We use

$N\left({ }^{13} \mathrm{CO}\right)=2.42 \times 10^{14} \frac{T_{\mathrm{ex}} \int \tau_{13} \mathrm{~d} v}{1-\exp \left(-5.29 / T_{\mathrm{ex}}\right)}$

to obtain the ${ }^{13} \mathrm{CO}$ column density. $\tau_{13}$ is the optical depth of the line, we assume that $T_{\mathrm{ex}}$ is $10 \mathrm{~K}$ and the ${ }^{13} \mathrm{CO}$ emission is optically thin. We use the relation $N\left(\mathrm{H}_{2}\right) / N\left({ }^{13} \mathrm{CO}\right) \sim 5 \times 10^{5}$ (e.g. Simon et al. 2001). Finally, we estimate a molecular mass and a

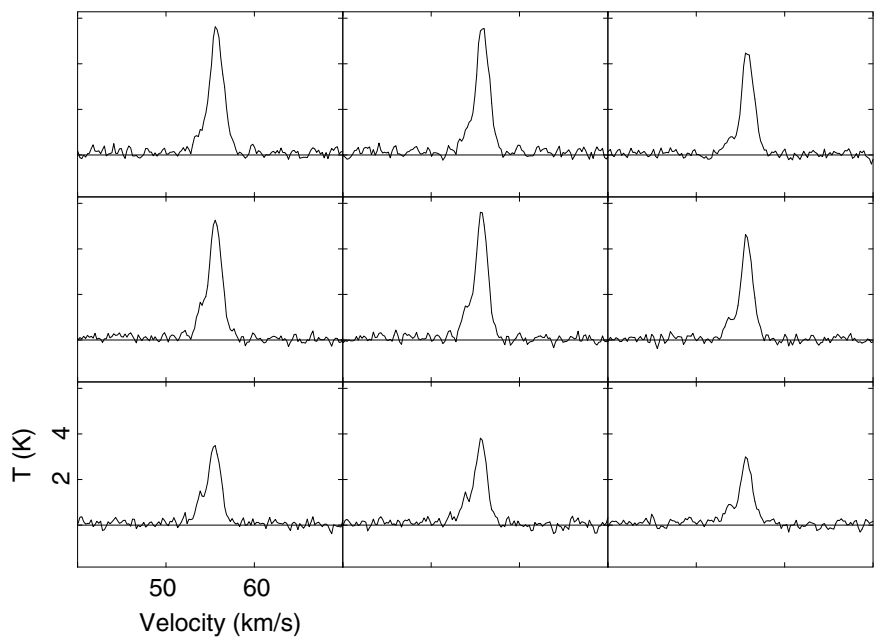

Fig. 2. ${ }^{13} \mathrm{CO} J=1-0$ spectra obtained towards the maximum of the molecular clump that lies over the SNR shell. The spectra, which show brightness temperature vs. velocity, correspond to nine positions observed in a region of about $50^{\prime \prime} \times 50^{\prime \prime}$ centered at the molecular clump peak.

density of $\sim 5 \times 10^{3} M_{\odot}$ and $\sim 500 \mathrm{~cm}^{-3}$, respectively, for each molecular clump. The mass value was obtained from

$$
M=\mu m_{\mathrm{H}} \sum\left[D^{2} \Omega N\left(\mathrm{H}_{2}\right)\right]
$$

where $\Omega$ is the solid angle subtended by the ${ }^{13} \mathrm{CO} J=1-0$ beam size, $m_{\mathrm{H}}$ is the hydrogen mass, while $\mu$, the mean molecular weight, is assumed to be 2.8 by taking into account a relative helium abundance of $25 \%$, and $D$ is the distance assumed to be $10.5 \mathrm{kpc}$. Summation was performed over all the observed positions within the $3.5 \mathrm{~K} \mathrm{~km} \mathrm{~s}^{-1}$ s contour level (not shown in Figs. 1 and 3 ).

We can estimate the required density matter in the $\gamma$-ray production region for hadrons from the observed $\gamma$-ray flux of HESS J1858+020. From

$\mathrm{d} F_{\gamma} / \mathrm{d} E\left(>E_{\min }\right)=N_{0}(E / 1 \mathrm{TeV})^{-\Gamma}$

we obtain $F_{\gamma} \sim 1.2 \times 10^{-12} \mathrm{~cm}^{-2} \mathrm{~s}^{-1}$. Here $E_{\min }=0.5 \mathrm{TeV}, \Gamma=$ 2.17, $N_{0}=0.6 \times 10^{-12} \mathrm{~cm}^{-2} \mathrm{~s}^{-1}$ (Aharonian et al. 2008a). Using Eq. (16) in Torres et al. (2003), we obtain a density of about $150 \mathrm{~cm}^{-3}$ assuming an acceleration efficiency of hadrons on the order of $3 \%$ and a supernova power of $10^{51} \mathrm{erg}$. Thus the molecular gas in positionally coincidence with HESS J1858+020 is densely enough to generate the very high energy emission hadronically.

The presence of at least two bright sources embedded in the southern molecular clump can be seen in Fig. 1 in the IR emission of the region studied here. The mid-IR emission also shows the presence of hot dust and policyclic aromatic hydrocarbons (PAHs), green and red in Fig. 1, respectively, which suggests an active star forming region. In order to confirm this, we carried out an IR photometric study of the sources that lie over the molecular clump that is centered at the HESS source position.

In Fig. 3 we present a three color image with three IRAC-Spitzer bands: red is $8 \mu \mathrm{m}$, green is $4.5 \mu \mathrm{m}$ and blue is $3.6 \mu \mathrm{m}$. As in Fig. 1 the white contours are the ${ }^{13} \mathrm{CO}$ $J=1-0$ emission integrated between 51 and $59 \mathrm{~km} \mathrm{~s}^{-1}$. To look for tracers of star formation activity, we use the GLIMPSE Point-Source Catalog to perform photometry. Considering only 


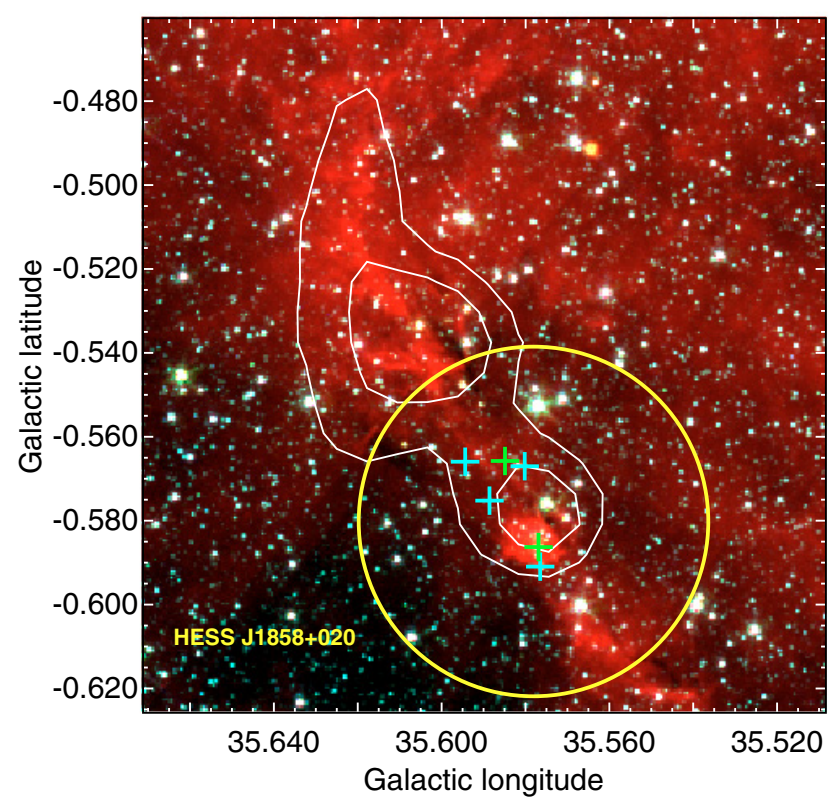

Fig. 3. Three color image of IRAC-Spitzer bands (red $=8 \mu \mathrm{m}$, green $=4.5 \mu \mathrm{m}$, and blue $=3.6 \mu \mathrm{m}$ ). The white contours are the ${ }^{13} \mathrm{CO}$ $J=1-0$ emission integrated between 51 and $59 \mathrm{~km} \mathrm{~s}^{-1}$, its levels are 3.2 and $5 \mathrm{~K} \mathrm{~km} \mathrm{~s}^{-1}$. The crosses are YSO candidates according to the photometric study described in the text. Green crosses are Class I sources and cyan crosses represent Class II sources.

sources that have been detected in the four Spitzer-IRAC bands, we found 32 sources within a circular area of $80^{\prime \prime}$ in radius, centered at the molecular clump. According to the criteria presented by Allen et al. (2004), we found six sources as young stellar object (YSO) candidates. Two of them are Class I objects (protostar with circumstellar envelope; green crosses in Fig. 3), and the others are Class II objects (young stars with disk-only emission; cyan crosses in Fig. 3). From Fig. 3 a bright source can be noticed that lies almost at the center of the molecular clump, but in this case we cannot perform photometry since it has some bands with null fluxes in the GLIMPSE catalog. However, from Fig. 3 it can be appreciated that this source appears slightly extended in the $4.5 \mu \mathrm{m}$ emission (green), which according to Cyganowski et al. (2008) may suggest that it could be a MYSO that drives outflows. For the sources that are also detected in the 2MASS $J H K$-bands, we derive their spectral energy distribution (SED) by fitting the fluxes using the tool developed by Robitaille et al. (2007), which is available online $^{1}$. This was possible only for three Class II sources, with the result that they are indeed young objects $\left(\sim 10^{5}\right.$ years). We conclude that this region is active in star formation, which can be considered as an alternative or complementary possibility to explain the very high energy emission. HESS J1858+020 could be a similar case as SNR W28. Several molecular clumps and star-forming regions were found towards W28 and proposed to be candidates to explain the very-high energy emission detected in the region (Aharonian et al. 2008b). Further sub-mm observations, high CO transitions and tracers of star formation will provide more accurate mass and density estimates and allow us to search for perturbed gas by both SNR shock and star formation processes.

\section{Summary}

In this work we investigate the ISM towards the southern border of the recently re-identified source G35.6-0.4 as a SNR, which coincides with the northern portion of the very high energy source HESS J1858+020. Analyzing the ${ }^{13} \mathrm{CO} J=1-0$ emission we find a molecular cloud in positional and kinematical agreement with the SNR, which suggests that the molecular gas is affected by the remnant shock. The discovered molecular cloud is composed by two clumps, each with a mass and density of $\sim 5 \times 10^{3} M_{\odot}$ and $\sim 500 \mathrm{~cm}^{-3}$, respectively. Considering the gamma-ray flux observed towards HESS J1858+020, we estimate that a molecular cloud with a density of at least $150 \mathrm{~cm}^{-3}$ could explain the very high energy emission hadronically. Thus we suggest that the interaction between the SNR G35.6-0.4 and the molecular cloud is responsible for the $\gamma$-ray emission. Additionally, analyzing mid-IR emission, we find that the region is active in star formation, which could be considered as an alternative or complementary possibility to explain the very high energy emission. This SNR-molecular cloud-HESS source complex could be a similar case as the high-energy sources detected towards SNR W28. Further sub-mm observations will provide more accurate mass and density estimates and allow us to search for perturbed gas by both SNR shock and star formation processes.

Acknowledgements. S.P. and E.G. are members of the Carrera del investigador científico of CONICET, Argentina. This work was partially supported by the CONICET grant PIP 112-200801-02166, UBACYT A023 and ANPCYT PICT2007-00902.

\section{References}

Aharonian, F. A., \& Atoyan, A. M. 1996, A\&A, 309, 917

Aharonian, F., Akhperjanian, A. G., Barres de Almeida, U., et al. 2008a, A\&A, 477,353

Aharonian, F., Akhperjanian, A. G., Bazer-Bachi, A. R., et al. 2008b, A\&A, 481, 401

Allen, L. E., Calvet, N., D’Alessio, P., et al. 2004, ApJS, 154, 363

Cyganowski, C. J., Whitney, B. A., Holden, E., et al. 2008, AJ, 136, 2391

Falgarone, E., Lis, D. C., Phillips, T. G., et al. 1994, ApJ, 436, 728

Fazio, G. G., Hora, J. L., Allen, L. E., et al. 2004, ApJS, 154, 10

Gabici, S., Aharonian, F. A., \& Blasi, P. 2007, Ap\&SS, 309, 365

Gabici, S., Aharonian, F. A., \& Casanova, S. 2009, MNRAS, 396, 1629

Green, D. A. 2009, MNRAS, 399, 177

Hinton, J. 2008, J. Phys. Conf. Ser., 110, 062011

Jackson, J. M., Rathborne, J. M., Shah, R. Y., et al. 2006, ApJS, 163, 145

Robitaille, T. P., Whitney, B. A., Indebetouw, R., \& Wood, K. 2007, ApJS, 169, 328

Simon, R., Jackson, J. M., Clemens, D. P., Bania, T. M., \& Heyer, M. H. 2001, ApJ, 551, 747

Stil, J. M., Taylor, A. R., Dickey, J. M., et al. 2006, AJ, 132, 1158

Torres, D. F., Romero, G. E., Dame, T. M., Combi, J. A., \& Butt, Y. M. 2003, Phys. Rep., 382, 303

Werner, M. W., Roellig, T. L., Low, F. J., et al. 2004, ApJS, 154, 1

Yamazaki, R., Kohri, K., Bamba, A., et al. 2006, MNRAS, 371, 1975 